OPEN ACCESS

Edited by:

Janarthanan Jayawickramarajah, Tulane University, United States

Reviewed by:

Milan Balaz,

Underwood International College, Yonsei University, South Korea Amparo Ruiz Carretero,

Centre National de la Recherche Scientifique (CNRS), France

*Correspondence: Hans-Achim Wagenknecht Wagenknecht@kit.edu

Specialty section: This article was submitted to

Supramolecular Chemistry, a section of the journal Frontiers in Chemistry

Received: 02 August 2019 Accepted: 17 September 2019 Published: 22 October 2019

Citation:

Fritz $Y$ and Wagenknecht $H$-A (2019) Influences of Linker and Nucleoside for the Helical Self-Assembly of Perylene Along DNA Templates. Front. Chem. 7:659. doi: 10.3389/fchem.2019.00659

\section{Influences of Linker and Nucleoside for the Helical Self-Assembly of Perylene Along DNA Templates}

\author{
Yannic Fritz and Hans-Achim Wagenknecht* \\ Karlsruhe Institute of Technology, Institute of Organic Chemistry, Karlsruhe, Germany
}

Six different conjugates of perylene with 2 '-deoxyuridine and with 2-amino-2'-deoxyadenosine were synthesized and applied for DNA-templated assembly in aqueous buffer solutions. They differ by the linkers ethynylene, phenylene, and phenylene-ethynylene between nucleoside and chromophore. The nucleosides were investigated as monomers in $\mathrm{CHCl}_{3}$ and dimethyl sulfoxide by optical spectroscopy. The properties of the four phenylene-linked conjugates are similar to that of perylene as reference because these linkers separate both aromatic parts. The ethynylene linker electronically couples the chromophore with the respective nucleoside and thus red shifts the absorbance. The DNA-templated assembly properties were elucidated by mixing the templates in aqueous buffer with the perylene-nucleoside conjugates from a dimethyl sulfoxide stock solution. Specific binding of the nucleosides was probed by comparing the results with $\mathrm{dA}_{20}$ and $\mathrm{T}_{20}$ as single-stranded DNA templates. Our studies reveal the structural parameters that are important for the DNA-templated assembly of perylenes. First, perylene-2'-deoxyuridine conjugates do not form DNA-templated helical assemblies, regardless of the choice of linker. Second, the ethynylene linker is crucial for successful DNA-templated chromophore assemblies of perylene-2-amino-2'-deoxyadenosine conjugates. Third, in contrast, the phenylene linker inhibits self-assembly along single-stranded DNA templates. In conclusion, the 2-amino-2'-deoxyadenosin in combination with the ethynylene linker provides the best structural feature for specific and helical DNA-templated assembly of perylenes. This result is important for the design of future DNA-based supramolecular architectures with chromophores, in particular DNA-based light-harvesting systems and DNA systems for emitting or sensing circularly polarized luminescence.

Keywords: DNA, chromophor, assembly, template, fluorescence

\section{INTRODUCTION}

Supramolecular chemistry and supramolecular polymerization summarize the efforts to self-organize molecules through interactions in a precise and controllable way. Probably more than any other class of compounds, DNA provides a rather unexplored and groundbreaking new access to this challenge via bottom-up, programmed, and hierarchically ordered assembly of molecules, in particular organic chromophores, into designed supramolecular DNA architectures. The last two centuries have shown that-beyond their pure biological purpose-nucleic acids are able to serve as unique structural basis to create nanometer-sized two- and three-dimensional objects. 

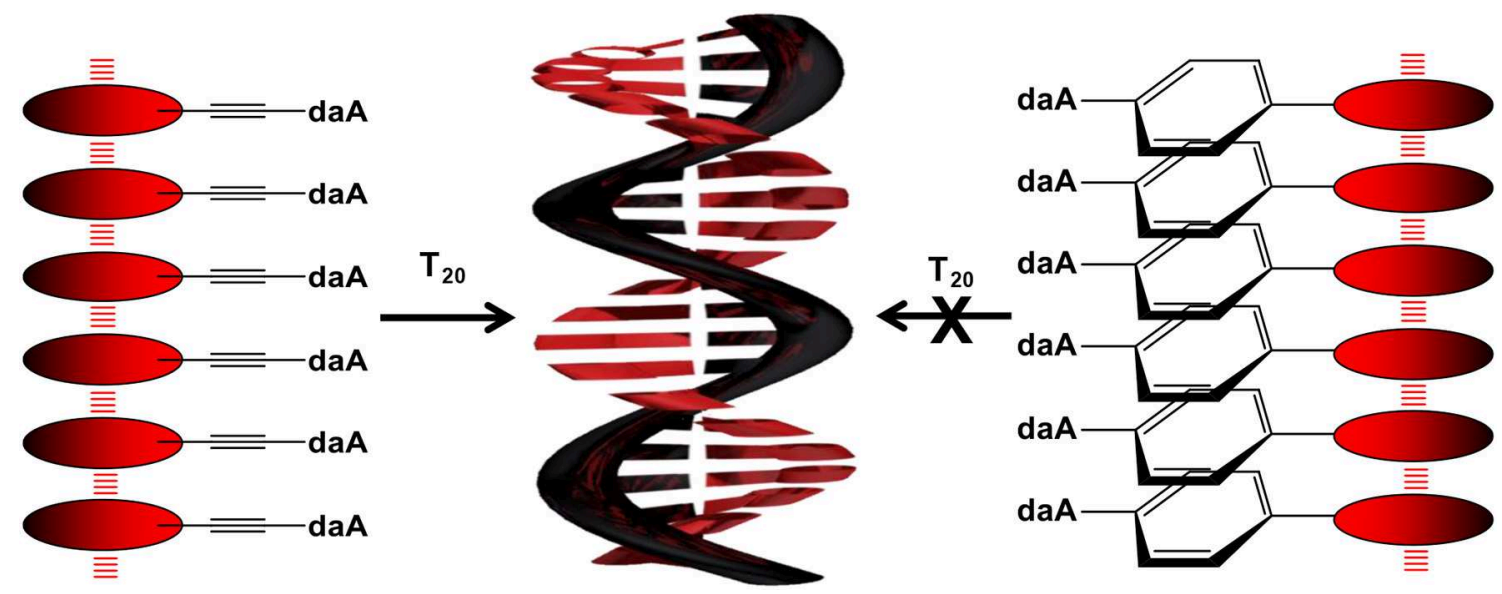

GRAPHICAL ABSTRACT | Illustration of the DNA-templated assembly of Pe-Et-daA and $\mathbf{P e - P h - d a A}$.

Representatively, the DNA nanostructures of Seeman (1982) and Kallenbach et al. (1983) and the DNA origami's by Rothemund (2006) are mentioned here. Since then, "DNA nanotechnology" has been established as the approach that applies self-assembly of DNA single strands to form molecular architectures in a highly organized manner (Yakovchuk et al., 2006; Lubrich et al., 2008; Wang et al., 2009; Chen et al., 2019). The sequence-defined recognition by canonical base pairing in DNA in combination with a perfectly coplanar stacking distance of $3.4 \AA$ and a helical chirality should yield supramolecular architectures (Burge et al., 2006). Such architectures are difficult to be achieved by simple organicchemical building blocks without DNA, neither covalently (polymers) nor non-covalently (supramolecular polymers). In a bottom-up approach, we follow herein the basic principle of chemistry research that "structure determines properties." Therefore, the controlled assembly of organic chromophores in supramolecular architectures based on nucleic acids holds the key potential for future functional materials with well-defined photochemical properties. In particular, the helical twist between the chromophores, which is induced by the DNA scaffold, controls electron transfer and energy transfer processes and thereby reduces the self-quenching that is typically observed in chromophore aggregates (Asanuma et al., 2003; Teo et al., 2009; Dutta et al., 2011; Kato et al., 2013; Li et al., 2013; Probst et al., 2014; Ishutkina et al., 2018).

In 2009, Kumar and Duff presented one of the first DNAbased light harvesting systems (Kumar and Duff, 2009). In the same year, our group published a temperature-controlled white-light-emitting DNA by covalent incorporation of pyrene- and Nile-red-modified nucleosides (Varghese and Wagenknecht, 2009). The combination with a perylenenucleoside yielded a light-harvesting antenna, and thereby, any excitation in the range between 350 and $600 \mathrm{~nm}$ efficiently resulted in a charge-separated state (Ensslen et al., 2015a). Two years later, the influence of the template strand length on the energy transfer of attached naphthalene nucleosides was investigated by the group of Stevens (Ruiz-Carretero et al., 2011; Stevens et al., 2011). Balaz et al. created nanoassemblies with porphyrine-linked 2-aminoadenines and oligothymidine as DNA templates, which showed an adjustable helicity depending on temperature gradients (Sargsyan et al., 2014). With pyrene and Nile red attached to different nucleosides, a sequence-specific self-assembly along a template DNA strand was accomplished in 2018 (Hofsass et al., 2018). Pyrene was linked via ethynylene bridge to 2-amino-2'-deoxyadenosine (daA) and Nile red to 2 '-deoxyuridine $(\mathrm{dU})$, which are complementary to thymine (T) and adenine (A), respectively, as recognition units of the template. Moreover, fullerene-DNA-chromophore assemblies were integrated as photoactive layers in solar cells that showed charge carrier generation in the spectral regime of all three components (Ensslen et al., 2016). These recent examples demonstrate the significance and potential of supramolecular DNA architectures.

To get a closer insight into the influence of linker and nucleoside on the optical and self-assembly properties with DNA, six different perylene-conjugated nucleosides (Figure 1) are presented herein that differ (i) by the linker between the chromophore and the nucleoside, including ethynylene (Et), phenylene ( $\mathrm{Ph})$, and the combined phenyleneethynylene (PhEt) linkers, and (ii) by the nucleoside, either $\mathrm{dU}$ that recognizes $\mathrm{A}$ in the DNA template, or daA that recognizes $\mathrm{T}$ in the DNA template. The Et linker yields a coplanar orientation of nucleoside and perylene parts in the conjugates, whereas the $\mathrm{Ph}$ linker introduces a rotational twist between the two parts. The PhEt linker combines the two extreme geometries and could potentially serve as compromise. We study the self-assembly of these nucleoside conjugates and the influence of the corresponding single-stranded DNA templates oligothymidine $\left(\mathbf{T}_{\mathbf{2 0}}\right)$ and oligo-2'-deoxyadenosine $\left(\mathbf{d A}_{\mathbf{2 0}}\right)$ in comparison by methods of optical spectroscopy to characterize their optical and chiroptical properties. 

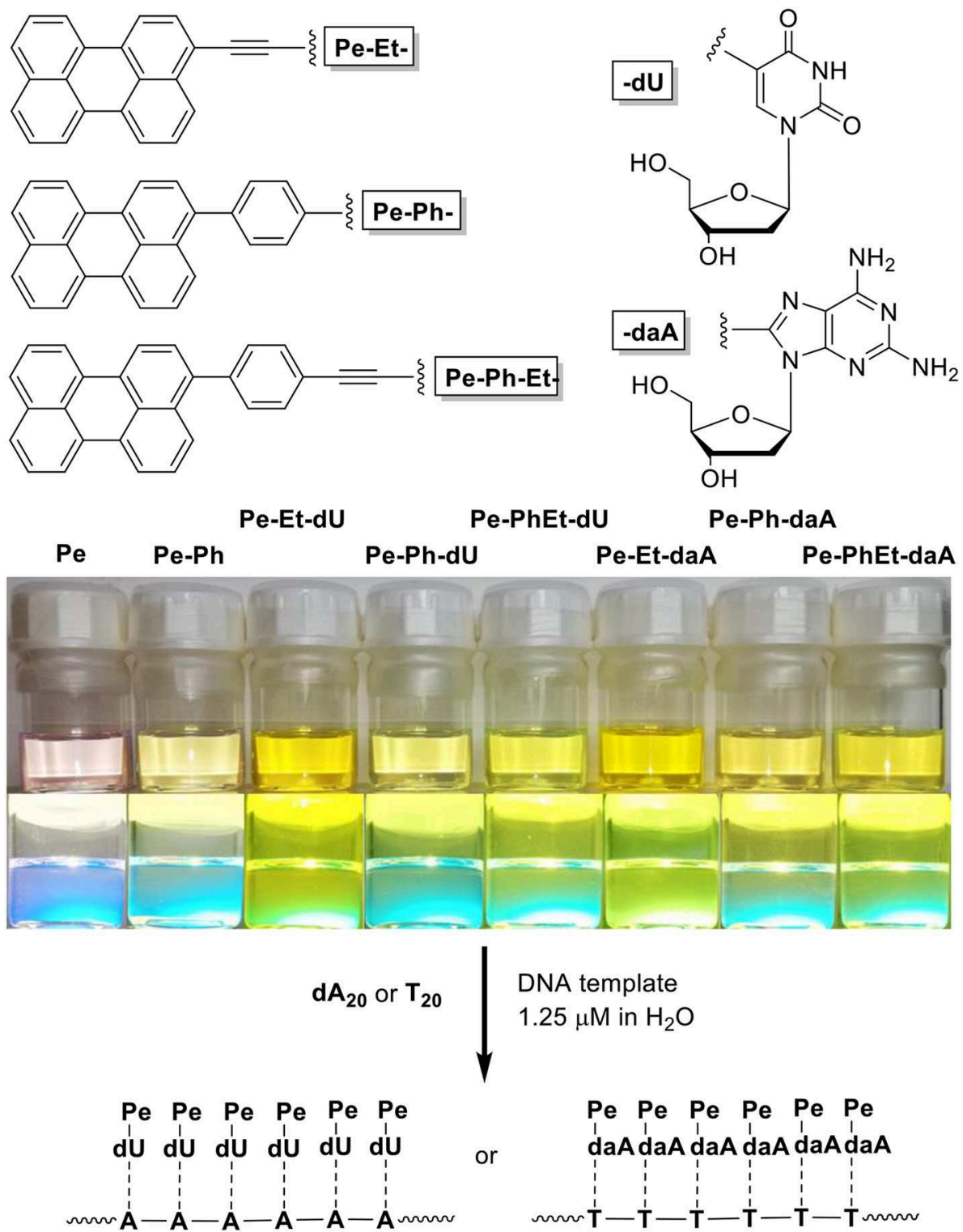

FIGURE 1 | (Top) Structure of the nucleoside conjugates Pe-Et-dU, Pe-Ph-dU, Pe-Ph-Et-dU, and Pe-Et-daA, Pe-Ph-daA, Pe-Ph-Et-daA. (Bottom) Image of solutions of perylene $(\mathbf{P e})$, 3-phenylperylene $(\mathbf{P e P h})$, and the nucleoside conjugates in $\mathrm{CHCl}_{3} /$ dimethyl sulfoxide $(\mathrm{DMSO})=4: 1(100 \mu \mathrm{M})$ and image of their fluorescence during excitation by a UV handheld lamp.

\section{MATERIALS AND METHODS}

All chemicals used for synthesis had at least the purification grade "for synthesis." Solvents used in synthesis, optical spectroscopy or analysis had the grade "HPLC" or "pro analysis." Water was deionized and ultra-filtrated by a Millipore Direct 8/16 from MERCK MILLIPORE. Unmodified DNA strands were bought from METABION, which were already HPLC-purified and lyophilized. The DNA was dissolved in water and concentrations were determined spectrometric with a NANODROP ND-100 spectrophotometer. All Pd-catalyzed reactions were performed under exclusion of oxygen and water (except for Suzuki-couplings, which used water as reagent). Reaction mixtures were treated by freezepump-thaw in three cycles before adding the catalyst or degassed with argon. Some reactions were performed in sealed glass vials $(10 \mathrm{~mL}$ or $20 \mathrm{~mL})$, which resulted in significantly higher yield compared to common round bottom flasks. The purity of all products were determined by NMR-spectroscopy and high-resolution mass spectrometry. NMR spectra were recorded on a BRUKER Advance 500 (500 MHz, 1H-NMR; $126 \mathrm{MHz}, 13 \mathrm{C}-\mathrm{NMR}$ ). Chemical shifts were reported in parts per million (ppm), relative to the standard tetramethylsilane $(\delta=0.00 \mathrm{ppm})$ and the spectrum was calibrated against the $1 \mathrm{H}$ residues of the deuterated 
solvents. Due to the bad solubility of most products, the use of deuterated pyridine was necessary. The mass-spectrometry was performed on a THERMOFISHER Scientientific Q Exactive (Orbitrap) by electron spray ionization (ESI) and reported in mass/charge $(\mathrm{m} / \mathrm{z})$. In case of daA-containing products, the protonated species was mostly found. For all spectroscopic experiments semi-micro quartz glass cuvettes from STARNA (width $10 \mathrm{~mm}$, volume $1.4 \mathrm{~mL}$ ) were used and all spectra were recorded at $20^{\circ} \mathrm{C}$. Absorption spectra were recorded on a Lambda 750 from PERKIN ELMER with a PTP-6+6 Peltier System. Circular dichroism was measured with a JASCO J810 Spectropolarimeter and the peltier-element PTC-423S (100 $\mathrm{nm} / \mathrm{min}, 4$ accumulations). Fluorescence was recorded on a Fluoromax-4 from HORIBA SCIENTIFIC with an AC 200 thermostat from THERMO SCIENTIFIC. All samples were excited at $420 \mathrm{~nm}$ and the spectra were divided by the absorbance at $420 \mathrm{~nm}$ for comparison. Absolute fluorescence quantum yields were determined with a Quantaurus QY C11347 from HAMAMATSU (measured in different concentrations between 40 and $60 \mu \mathrm{M}$ and averaged). Self-assembly experiments were prepared as follows: Chromophore $45 \mathrm{mM}$, DNA template $1.5 \mu \mathrm{M}$, phosphate-buffer $10 \mathrm{mM}$ (pH 7.0), $250 \mathrm{mM} \mathrm{NaCl}$. Due to solubility issues, the chromophores were added from a $1 \mathrm{mM}$ stock solution in DMSO, which causes a DMSO content of $4.5 \%$. For CD and fluorescence experiments the Chromophore was added shortly before the measurement, to minimize the influence of precipitation.

\section{RESULTS AND DISCUSSION}

All nucleoside conjugates were synthesized as described in the Supporting Information. The key steps are Pd-catalyzed Sonogashira and Suzuki couplings between the halogenated nucleoside precursors and the perylene derivatives. We expected that the linker between chromophore and nucleoside has a strong impact on the photophysical properties. In principal, the ethynylene group connects the perylene chromophore with the nucleosides in a coplanar orientation and thereby yields $\pi$-conjugation between them, whereas the phenylene group electronically isolates the $\pi$-systems by rotation. Indeed, this structural influence is clearly observable by the ultraviolet-visible (UV/vis) absorbance (Figure 2): The characteristic perylene absorbance between 400 and $520 \mathrm{~nm}$ is nearly identical for all $\mathrm{Ph}$ - and $\mathrm{Ph}$-Et-linked conjugates compared to 1-phenyl-perylene as reference (Kawasumi et al., 2013). In particular, both $\mathbf{P e - P h -}$ dU and $\mathbf{P e}-\mathbf{P h}-\mathbf{d a A}$ show only very little absorbance differences to the reference, which is an advantage of the $\mathrm{Ph}$ linker that efficiently interrupts the $\pi$-conjugation with the nucleoside. Only the Et-linked conjugates Pe-Et-dU and Pe-Et-daA display an absorbance that is red shifted by 23 and $35 \mathrm{~nm}$, respectively. This shows the $\pi$-conjugating effect of the Et linker.

In order to study the effect of the nucleosides and the linkers on the fluorescence, the spectra and quantum yields were recorded (Table 1 and Figure $\mathbf{S 1}$ ). In $\mathrm{CHCl}_{3}$ as solvent [with $6 \%$ dimethyl sulfoxide (DMSO) to ensure solubility], the four $\mathrm{Ph}$ - and $\mathrm{Ph}$-Et-conjugated nucleosides yield higher quantum yields (77-84\% for $\mathrm{dU}$ and $83 \%$ for daA conjugates) than $\mathbf{P e}$ Et-dU and Pe-Et-daA (58 and 78\%, respectively). These results agree with the electronically separating character of the Ph linker, too. In contrast, the Et group enlarges the $\pi$-system and thus lowers the quantum yields. In conclusion, these results show the

TABLE 1 | Fluorescence quantum yields of perylene (Pe), 1-phenylperylene (PePh), and the perylene-nucleosides in DMSO and $\mathrm{CHCl}_{3}$ with $6 \% \mathrm{DMSO}$ (average of 40,50 , and $60 \mu \mathrm{M}$ ), $\lambda_{\mathrm{exc}}=420 \mathrm{~nm}$.

\begin{tabular}{|c|c|c|}
\hline Nucleoside & $\Phi_{\mathrm{F}}$ in DMSO & $\Phi_{\mathrm{F}}$ in $\mathrm{CHCl}_{3}(6 \%$ DMSO) \\
\hline Pe-Et-dU & $76 \pm 0.97 \%$ & $58 \pm 1.1 \%$ \\
\hline Pe-Ph-dU & $96 \pm 1.9 \%$ & $84 \pm 0.16 \%$ \\
\hline Pe-Ph-Et-dU & $90 \pm 1.6 \%$ & $77 \pm 0.45 \%$ \\
\hline Pe-Et-daA & $6.7 \pm 0.21 \%$ & $78 \pm 0.66 \%$ \\
\hline$P e-P h-d a A$ & $2.8 \pm 0.08 \%$ & $83 \pm 0.21 \%$ \\
\hline Pe-Ph-Et-daA & $5.5 \pm 0.16 \%$ & $83 \pm 0.21 \%$ \\
\hline $\mathrm{Pe}$ & $\sim 100 \%$ & $98 \pm 0.60 \%$ \\
\hline $\mathrm{PePh}$ & $\sim 100 \%$ & $\sim 100 \%$ \\
\hline
\end{tabular}
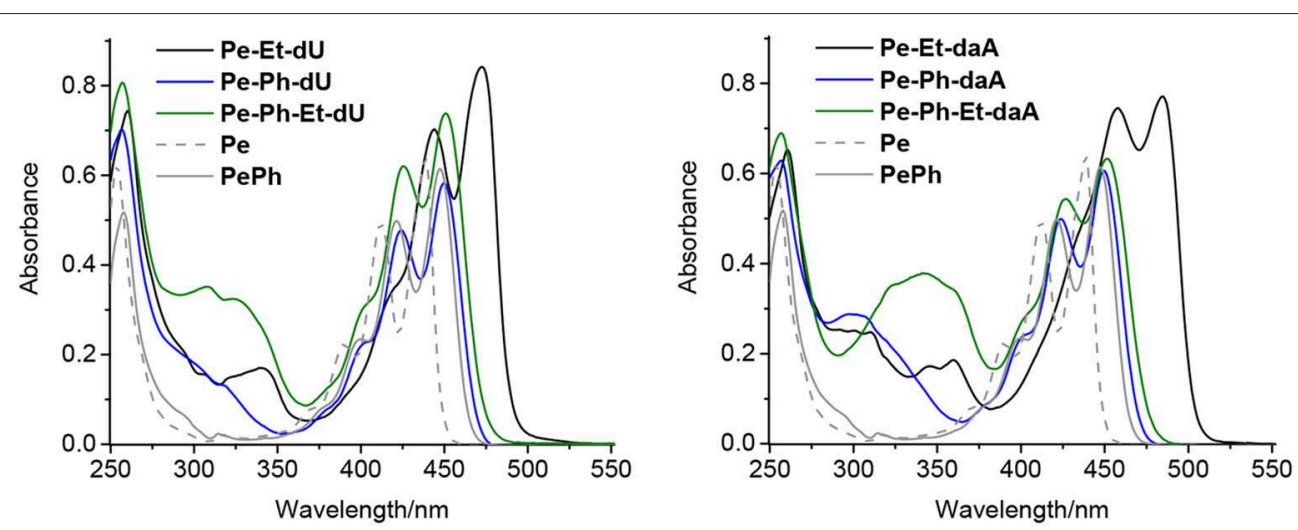

FIGURE 2 | Ultraviolet-visible (UV/vis) absorption spectra of perylene (Pe), 1-phenylperylene (PePh), and the perylene-nucleosides with dU (Left) and with daA (Right), each $20 \mu \mathrm{M}$ in $\mathrm{CHCl}_{3}$ [2\% dimethyl sulfoxide (DMSO)]. 
expected tuning of the optical properties of the chromophorenucleoside conjugates by the choice of linker. Surprisingly, the emissions of the daA-modified chromophores are nearly quantitatively quenched in pure DMSO, while the emissions of the dU-modified ones give quantum yields from 76 to $96 \%$. These striking fluorescence quantum yield differences in DMSO become plausible by photoinduced electron transfer in the daA conjugates that occurs only in the highly polar solvent DMSO. Based on redox potentials from literature, daA can be more easily oxidized ( $E_{\mathrm{OX}}=1.1 \mathrm{~V}$ vs. NHE in water) (Stockert et al., 2002) than $\mathrm{dU}\left(E_{\mathrm{ox}}=1.34 \mathrm{~V}\right)$ (Faraggi et al., 1996). Using the reduction potential for perylene of $E_{\text {red }}=-1.91 \mathrm{~V}$ together with $E_{00}=2.8 \mathrm{eV}$ (Rossetti et al., 2005), the Rehm-Weller equation for the driving forces $\Delta G=E_{\mathrm{ox}}-E_{\text {red }}-E_{00}+E_{\mathrm{C}}$ (Coulomb energy $E_{\mathrm{c}}$ ) gives a small $\Delta G$ of $-0.3 \mathrm{eV}$ for the daA conjugates in favor of a photoinduced charge transfer, but a negligible $\Delta G$ for the $\mathrm{dU}$ conjugates (without consideration of $E_{\mathrm{C}}$ ) that makes the photoinduced charge transfer very unlikely in the latter conjugates. Obviously, the different polarity of the solvents DMSO and $\mathrm{CHCl}_{3}$ influence the Coulomb energy in the chargeseparate state such that only in the highly polar solvent DMSO the photoinduced electron transfer in the daA conjugates is observable. In DMSO, the charge-separated state is stabilized and thereby the Coulomb energy is reduced.

To examine the self-assembling ability of the nucleoside conjugates, experiments with $\mathbf{T}_{\mathbf{2 0}}$ and $\mathbf{d A}_{\mathbf{2 0}}$, respectively, as single-stranded DNA templates were performed. The chromophore conjugates were characterized by their UV/vis absorbance, fluorescence, and circular dichroism in aqueous buffer at $\mathrm{pH} 7$ to elucidate (i) their self-assembling properties and, more importantly (ii) the potential influence of the singlestranded DNA templates. Specific binding of the nucleoside conjugates was elucidated by comparing the results with $\mathbf{d A}_{20}$ and $\mathbf{T}_{\mathbf{2 0}}$ as templates. Previous work in our group revealed that the assembly of nucleosides along DNA templates succeeded at room temperature with no annealing (Hofsass et al., 2018). We kept this method for the studies herein. The shapes of all absorption spectra in $\mathrm{H}_{2} \mathrm{O}$ differ from those of the monomer reference spectra in DMSO. First, the absorbance of the assemblies of Pe-Et-dU (Figure 3, left) and Pe-Et-daA (Figure 3, middle) along the DNA templates were compared to DNA-free samples in aqueous buffer (with 4.5\% DMSO from the perylene-nucleoside stock solutions). In case of Pe-Et-dU, the perylene-typical fine structure is significantly broadened, indicating self-assembly of the chromophores. There is only little influence of the complementary DNA template $\mathbf{d A}_{\mathbf{2 0}}$, if at all, by a slightly increased absorbance compared to the non-complementary template $\mathbf{T}_{\mathbf{2 0}}$ and compared to the absence of any DNA, too. For Pe-Et-daA in the presence of the complementary template $\mathbf{T}_{\mathbf{2 0}}$, however, the perylene-typical fine structure is maintained but with an altered ratio of the included absorption bands. With the non-complementary template $\mathbf{d A}_{\mathbf{2 0}}$ and without any DNA, this fine structure is completely lost, and the absorbance is significantly blue shifted to a broad absorbance from 350 to $550 \mathrm{~nm}$ with a maximum at $408 \mathrm{~nm}$. Since this absorbance is similar to that without the template, we assign it to the non-templated self-assembly of Pe-Et-daA. The influence of the complementary DNA template $\mathbf{T}_{\mathbf{2 0}}$ is significant and serves as first indication for a base pairing of the Pe-Et-daA monomers along this template and thus the formation of an ordered and helical assembly (vide infra). It is important to mention here that daA is, in principal, a modified 2 -deoxyadenosine with a second amino group to enhance the binding selectivity by a potential third hydrogen bond to thymidines as recognition unit in the DNA template $\mathbf{T}_{\mathbf{2 0}}$ (Hofsass et al., 2018). The lacking third hydrogen bond between Pe-Et-dU and the DNA templates probably accounts for its lacking selectivity between $\mathbf{d A}_{\mathbf{2 0}}$ and $\mathbf{T}_{\mathbf{2 0}}$ (and
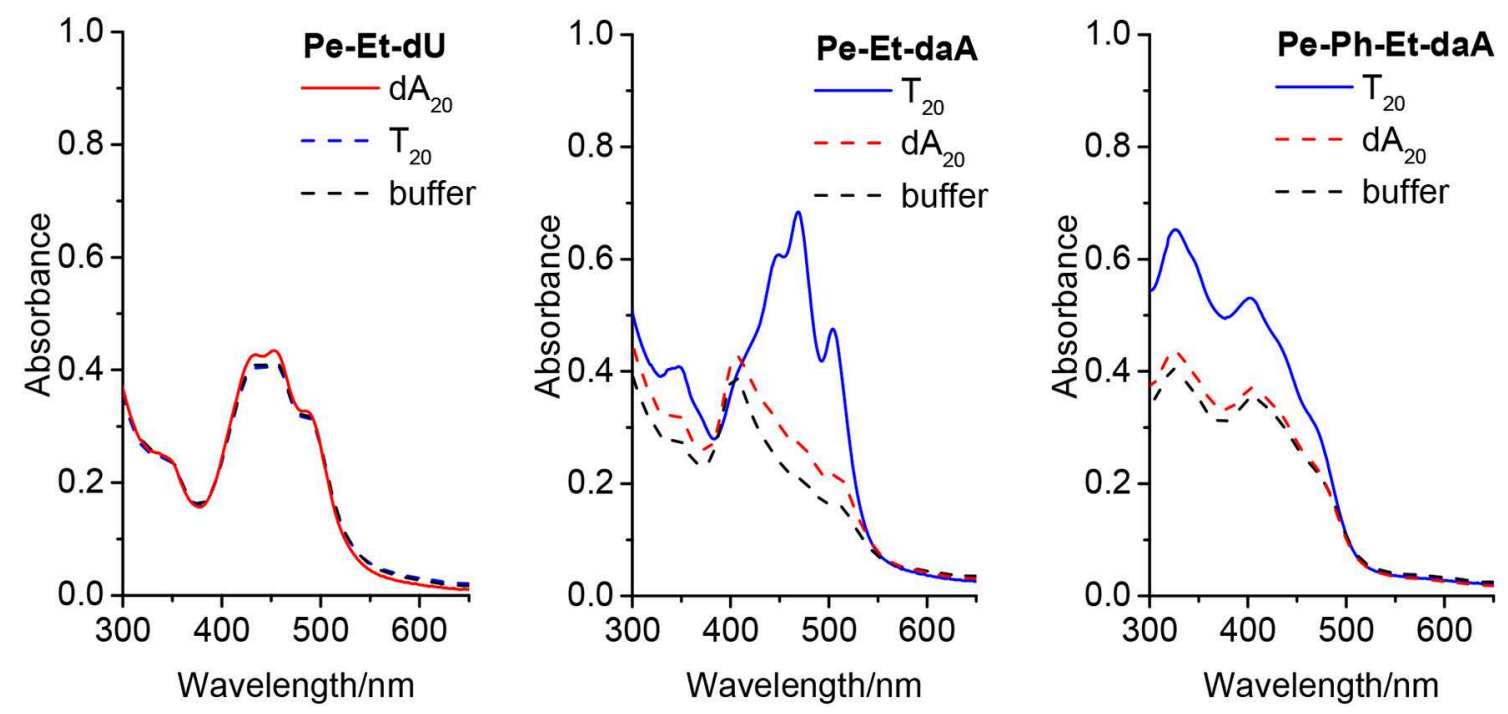

FIGURE 3 | Ultraviolet-visible (UV/vis) absorbance of Pe-Et-dU (Left), Pe-Et-daA (Middle), and Pe-Ph-Et-daA (Right), each $45 \mu$ M in aqueous buffer (10 mM sodium phosphate, $\mathrm{pH} 7,250 \mathrm{mM} \mathrm{NaCl}$, with 4.5\% DMSO) without and with DNA templates $\mathbf{T}_{\mathbf{2 0}}$ and $\mathbf{d} \mathbf{A}_{\mathbf{2 0}}(1.5 \mu \mathrm{M})$, respectively. 
without template). The UV/vis absorbances of the remaining four nucleosides with $\mathrm{Ph}$ - and $\mathrm{Ph}-\mathrm{Et}$ linkers in water look all similar and do not allow to elucidate any influence of the single-stranded DNA templates on the formation of these chromophore assemblies (Figure 3, right and Figure S3). This indicates that the $\mathrm{Ph}$ linkers are not all suitable for DNA-templated assembly of perylenes. A careful look on the absorbances reveals, however, that only Pe-Ph-Et-daA shows an enhanced absorbance at $408 \mathrm{~nm}$ in the presence of the complementary template $\mathbf{T}_{\mathbf{2 0}}$ compared to $\mathbf{d A}_{\mathbf{2 0}}$ and to pure buffer (Figure 3, right), indicating that there might be a small structural influence of the DNA on this particular chromophore assembly.

The fluorescence further supports the elucidation of structural prerequisites for the assembly of perylene-nucleoside conjugates along single-stranded DNA templates that were indicated by their UV/vis absorbance. In all non-templated samples, an unstructured and broad fluorescence was observed at $\sim 600$ $650 \mathrm{~nm}$, which we assign to perylene excimers as a result of the self-assembly of the perylene-nucleoside conjugates in water. In particular, both Pe-Et-dU and Pe-Et-daA without DNA templates show this unstructured and broad fluorescence at 600 and $650 \mathrm{~nm}$, respectively (Figure 4, left and middle). If we assume a coplanar arrangement by $\pi-\pi$ stacking between the perylene monomers and Watson-Crick or any similar base pairing between the nucleoside conjugates and the templates, a helical twist is introduced by the DNA to the perylene assembly in water. According to our previous results with pyrene conjugates, such helicity typically reduces excimer-like fluorescence (Trifonov et al., 2005). In fact, the assembly in the presence of the template $\mathbf{T}_{\mathbf{2 0}}$ significantly changes the fluorescence readout of Pe-Et-daA. This sample shows mainly a blue-shifted and enhanced fluorescence at $590 \mathrm{~nm}$, which we assign to the ordered and presumably helical assembly of these chromophores. This assignment supports the considerably different absorbance of $\mathbf{P e - E t - d a A ~ w i t h ~} \mathbf{T}_{\mathbf{2 0}}$ as described above. The assembly of Pe-Et-dU shows only fluorescence between 450 and $550 \mathrm{~nm}$ and only little excimer fluorescence intensity. The lacking influence of neither $\mathbf{d A}_{\mathbf{2 0}}$ nor $\mathbf{T}_{\mathbf{2 0}}$ as complementary or non-complementary templates on the fluorescence readout implies that Pe-Et-dU forms a stable non-templated selfassembly. This stands in contrast to Pe-Et-daA for which only the complementary DNA template $\mathbf{T}_{\mathbf{2}}$ orders the chromophore assembly in such a way that the fluorescence is blue shifted. In contrast to the two Et-linked nucleosides, the fluorescence of $\mathbf{P e}-$ $\mathbf{P h}-\mathbf{d U}, \mathbf{P e}-\mathbf{P h}-\mathbf{d a A}$, and Pe-Ph-Et-dU show only excimer-like fluorescence of the non-templated and self-assembled perylene conjugates at $650 \mathrm{~nm}$, which is not significantly altered by the DNA templates (Figure S2). Similar to the observations by UV/vis absorption spectroscopy, the DNA templates have no significant effect on the self-assembly of the $\mathrm{Pe}-\mathrm{Ph}$ conjugates. Obviously, the twist introduced by the $\mathrm{Ph}$ in these nucleoside conjugates disturbs the coplanar orientation of nucleoside and perylene and thereby inhibits the templated assembly along the single-stranded DNA. Typical DNA double helix has a stacking distance of $0.34 \mathrm{~nm}$ between two base pairs, whereas the diameter of $\mathrm{Ph}$ is $0.43 \mathrm{~nm}$; a rotational twist in the $\mathrm{Ph}$ linked conjugates cannot be accepted by this type of assembly. A careful look onto the fluorescence of Pe-Ph-Et-daA reveals a small blue shift from $628 \mathrm{~nm}$ (with the non-complementary template $\mathbf{d A}_{\mathbf{2 0}}$ and without any template) to $618 \mathrm{~nm}$ with the complementary template $\mathbf{T}_{\mathbf{2 0}}$ (Figure $\mathbf{4}$, right) and thereby supports the small influence of this DNA template on this particular chromophore assembly similarly to the previously discussed UV/vis absorbance.

To get a closer look into the possible helical orientations of the perylene-nucleoside conjugates introduced by the singlestranded DNA template, circular dichroism (CD) was recorded for all samples. We do not interpret the $\mathrm{CD}$ in the range between 250 and $350 \mathrm{~nm}$ because the perylene-nucleoside absorbance overlaps with the template absorbance. All nucleoside conjugates without DNA template display only small chiroptical activity in DMSO but no sign for ordered helical arrangements (Figure S4). These signals mainly arise from the chromophores connected to the chiral $\beta$-D-ribofuranoside residue and in particular in DMSO as good solvent for both parts of the nucleoside conjugates, polar and non-polar. The CD spectra of Pe-Et-dU with $\mathbf{d A}_{\mathbf{2 0}}$, with $\mathbf{T}_{\mathbf{2 0}}$ and without any DNA template are similar to those of the monomers in DMSO (Figure 5, left). This result further supports our explanation that the stable, non-templated self-assemblies of Pe-Et-dU are not chiral and they are not significantly influenced by the DNA template. The intrinsic self-stacking of the perylenes overrules any template effect. Similarly, small CD signals are observed in the samples with $\mathbf{P e}-\mathbf{P h}-\mathbf{d U}, \mathbf{P e}-\mathbf{P h}-$ daA, and Pe-Ph-Et-dU (Figure S5). This makes conclusively clear that two potential hydrogen bonds, which are provided by the dU unit, are not sufficient for effective and specific assembly of perylenes along DNA templates. In contrast, both Pe-Et$\mathbf{d a A}$ and Pe-PhEt-daA show strong chiroptical signals, which are enhanced in presence of $\mathbf{T}_{\mathbf{2 0}}$. The CD of Pe-Et-daA with $\mathbf{T}_{\mathbf{2 0}}$ (Figure $\mathbf{5}$, middle) displays a clearly excitonically coupled signal in the perylene absorbance range between 350 and $550 \mathrm{~nm}$ by the combination of a negative Cotton effect followed by a positive Cotton effect. This indicates a left-handed chirality in these DNA-based assemblies with coplanarily stacked Pe-EtdaA chromophores. We previously observed this chirality for DNA-templated assemblies with pyrene- and Nile-red-modified nucleosides (Ensslen et al., 2015b; Hofsass et al., 2018). Even stronger $\mathrm{CD}$ signals were observed for the templated assemblies of Pe-Ph-Et-daA with $\mathbf{T}_{20}$ (Figure 5, right) but not for $\mathbf{P e - P h}-$ Et-dU with $\mathbf{d A}_{20}$ (Figure S5). Obviously, the Ph-Et linker still allows the chromophore assembly along $\mathbf{T}_{\mathbf{2 0}}$, but the perylene orientation differs from that of the clearly excitonically couples and coplanarily stacked Pe-Et-daA assembly with $\mathbf{T}_{\mathbf{2 0}}$ because the $\mathrm{CD}$ shows only a negative Cotton effect. Overall, there are two major results with respect to the structural parameters: (i) The structural influence of the DNA template is observed only with the daA conjugates but not with the $\mathrm{dU}$ conjugates, and (ii) while the Et-linked chromophores with daA selectively assemble along the complementary template strand with lefthanded chirality, the Ph-linked chromophores do not form templated water-soluble assemblies. Only the conjugate of daA 

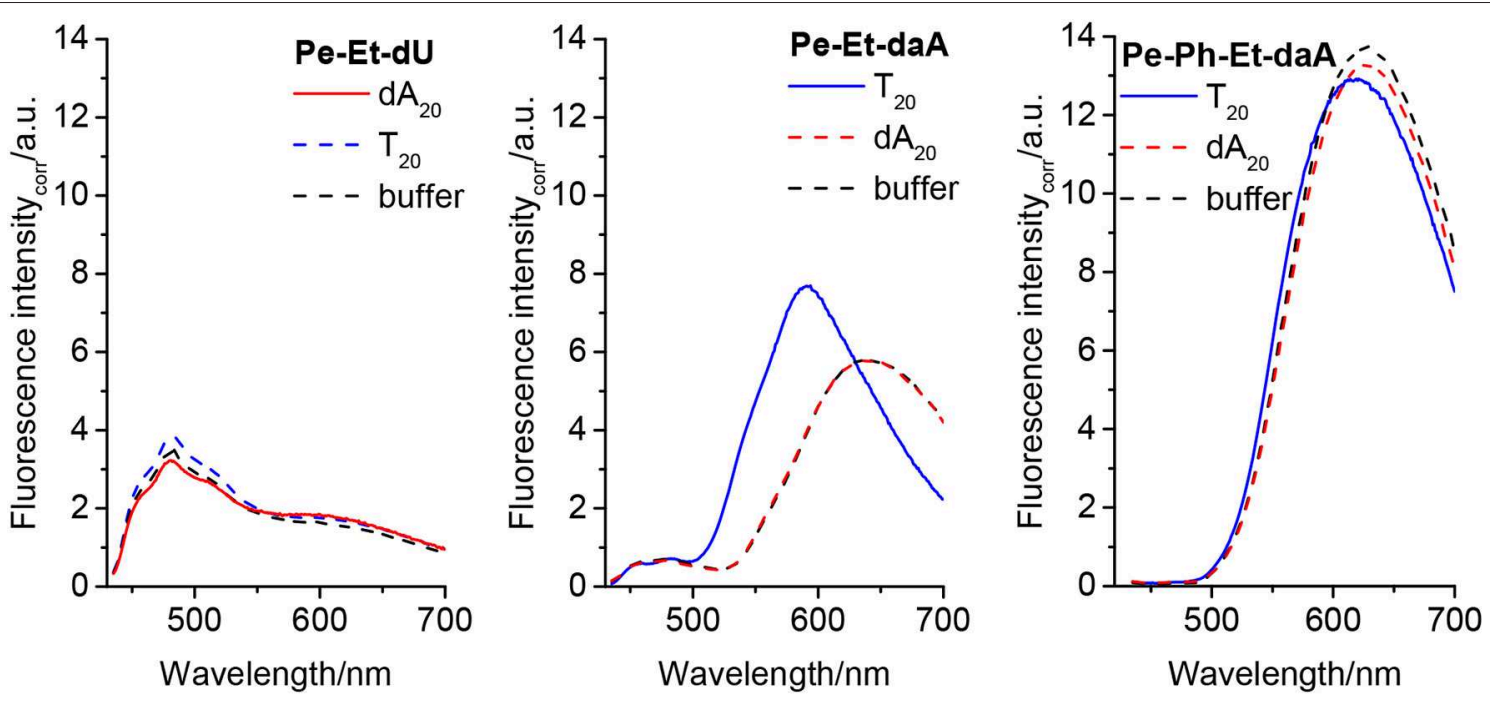

FIGURE 4 | Fluorescence of Pe-Et-dU (Left), Pe-Et-daA (Middle), and Pe-Et-daA (Right), each $45 \mu \mathrm{M}$ in aqueous buffer (10 mM sodium phosphate, pH 7 , $250 \mathrm{mM} \mathrm{NaCl}$, with $4.5 \% \mathrm{DMSO})$ without and with DNA templates $\mathbf{T}_{\mathbf{2 0}}$ and $\mathbf{d} \mathbf{A}_{\mathbf{2 0}}(1.5 \mu \mathrm{M})$, respectively. Excitation at $420 \mathrm{~nm}$. The fluorescence intensity was corrected by the absorbance at $420 \mathrm{~nm}$.
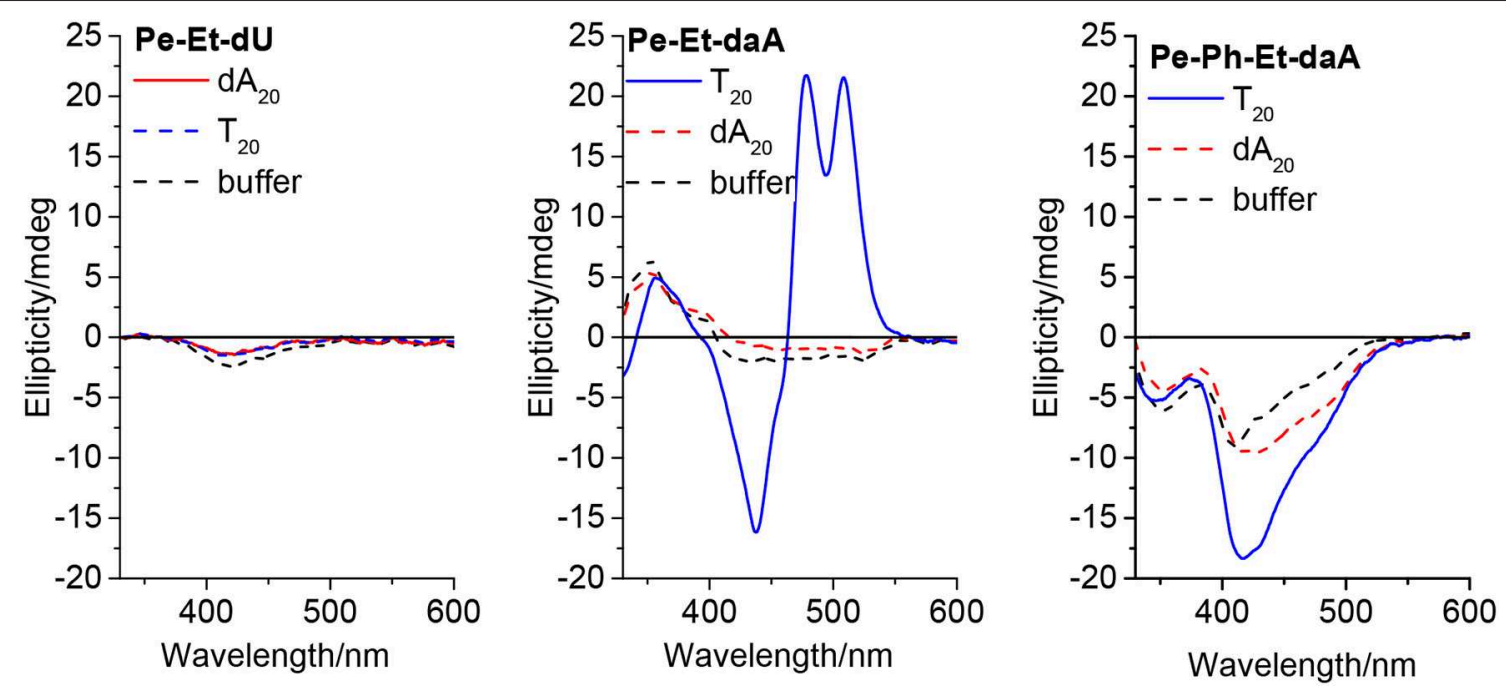

FIGURE 5 | Circular dichroism of Pe-Et-dU (Left), Pe-Et-daA (Middle), and Pe-Ph-Et-daA (Right), each $45 \mu$ M in aqueous buffer (10 mM sodium phosphate, $\mathrm{pH} 7,250 \mathrm{mM} \mathrm{NaCl}$, with $4.5 \%$ DMSO) without and with DNA templates $\mathbf{T}_{20}$ and $\mathbf{d A}_{\mathbf{2 0}}(1.5 \mu \mathrm{M})$, respectively.

with both linkers (Ph-Et) interact with the DNA templates in a different type of chiral assembly.

\section{CONCLUSIONS}

Six different conjugates of perylene with $\mathrm{dU}$ and with daA were synthesized and applied for DNA-templated assembly in aqueous solutions. The perylene conjugates differ by the linkers between the perylene chromophore and by the aromatic nucleoside heterocycle. The photophysical properties of the nucleoside monomers and their self-assemblies were investigated by optical spectroscopy methods. The $\mathrm{Ph}$ linker as well as the combined $\mathrm{Ph}-\mathrm{Et}$ linker separate both aromatic parts. Accordingly, the optical properties of the $\mathrm{Pe}-\mathrm{Ph}$ and $\mathrm{Pe}-\mathrm{Ph}-\mathrm{Et}$ conjugates with $\mathrm{dU}$ and daA are similar to that of perylene and 1phenylperylene as reference chromophores. In comparison, the Et linker electronically couples the chromophores and thus red shifts the absorbance. The DNA assemblies were formed by mixing the templates in water with the perylene-nucleoside conjugates from a DMSO stock solution. Important structural 
parameters for the DNA-templated assembly of perylenes were elucidated: (i) Perylene-dU conjugates do form stable, nontemplated assemblies and overrule the DNA template effect. $\mathrm{dU}$ can base pair only by two hydrogen bonds with the nucleotides in the DNA template, whereas daA provides the donors and acceptor for three hydrogen bonds. Although we do not provide direct experimental evidenced for base pairing via hydrogen bonding similar to Watson-Crick base pairing, this difference seems to be striking in particular for the comparison of the assemblies with Pe-Et-dU vs. with PeEt-daA. (ii) The Et linker is required for successful DNAtemplated chromophore assemblies of the conjugate with daA. It orientates both aromatic parts in a coplanar orientation. Obviously, this facilitates the formation of a helical DNAtemplated assembly with presumably $\pi-\pi$-stacked coplanarily arranged perylenes, which was evidenced in particular by the assemblies of $\mathbf{P e - E t - d a A ~ i n ~ c o m p a r i s o n ~ w i t h ~} \mathbf{P e}-\mathbf{P h}-\mathbf{d a A}$. (iii) The $\mathrm{Ph}$ linker twists both aromatic parts and thus completely inhibits self-assembly along single-stranded DNA templates in aqueous solutions. Accordingly, the conjugate Pe-Et-daA shows the strongest selectivity to the complementary DNA templates, while the Ph-linked one did not form templated assemblies. The assembly of Pe-Et-daA with $\mathbf{T}_{\mathbf{2 0}}$ shows a left-handed chirality and excitonic coupling. The conjugate Pe-Ph-Et-daA with both linkers shows also a structural influence by $\mathbf{T}_{20}$ as DNA template in particular according to the chiroptical properties; however, the structure of this perylene assembly significantly differs from the coplanar and excitonically coupled Py-Et-daA assembly. Overall, the daA nucleoside in combination with the Et linker provides the two structural prerequisites for specific and helical DNA-templated assembly that were identified by our study. This result is in agreement with other DNA-templated

\section{REFERENCES}

Asanuma, H., Kashida, H., Liang, X., and Komiyama, M. (2003). DNA-Naphthyl Red conjugate as a visualizing probe of DNA hybridization. Chem. Commun. 1536-1537. doi: 10.1039/B302875E

Burge, S., Parkinson, G. N., Hazel, P., Todd, A. K., and Neidle, S. (2006). Quadruplex DNA: sequence, topology and structure. Nucl. Acids Res. 34, 5402-5415. doi: 10.1093/nar/gkl655

Chen, K., Kong, J., Zhu, J., Ermann, N., Predki, P., and Keyser, U. F. (2019). Digital data storage using DNA nanostructures and solidstate nanopores. Nano Lett. 19, 1210-1215. doi: 10.1021/acs.nanolett. $8 \mathrm{~b} 04715$

Dutta, P. K., Varghese, R., Nangreave, J., Lin, S., Yan, H., and Liu, Y. (2011). DNA-directed artificial light-harvesting antenna. J. Am. Chem. Soc. 133, 11985-11993. doi: 10.1021/ja1115138

Ensslen, P., Brandl, F., Sezi, S., Varghese, R., Kutta, R. J., Dick, B., et al. (2015a). DNA-based oligochromophores as light-harvesting systems. Chem. Eur. J. 21, 9349-9354. doi: 10.1002/chem.201501213

Ensslen, P., Fritz, Y., and Wagenknecht, H. A. (2015b). Mixed noncovalent assemblies of ethynyl nile red and ethynyl pyrene along oligonucleotide templates. Org. Biomol. Chem. 13, 487-492. doi: 10.1039/C4OB $01860 \mathrm{E}$

Ensslen, P., Gartner, S., Glaser, K., Colsmann, A., and Wagenknecht, H. A. (2016). A DNA-fullerene conjugate as a template for supramolecular chromophore assemblies: towards DNA-based solar cells. Angew. Chem. Int. Ed. Engl. 55, 1904-1908. doi: 10.1002/anie.201509332 chromophore arrangements by us and others (Stevens et al., 2011; Sargsyan et al., 2014; Hofsass et al., 2018). Such investigations are important for the design of DNA-based supramolecular architectures with chromophores, in particular DNA-based lightharvesting systems and DNA architectures for emitting or sensing circularly polarized luminescence.

\section{DATA AVAILABILITY STATEMENT}

All datasets generated for this study are included in the manuscript/Supplementary Files.

\section{AUTHOR CONTRIBUTIONS}

YF did all experiments and wrote parts of the manuscript. H-AW supervised the research and wrote the manuscript.

\section{ACKNOWLEDGMENTS}

Financial support by the Deutsche Forschungsgemeinschaft (Wa $1386 / 20-1$ ) and KIT was gratefully acknowledged.

\section{EXPERIMENTAL PART}

All experimental procedures are described in the Supporting Information.

\section{SUPPLEMENTARY MATERIAL}

The Supplementary Material for this article can be found online at: https://www.frontiersin.org/articles/10.3389/fchem. 2019.00659/full\#supplementary-material

Faraggi, M., Broitman, F., Trent, J. B., and Klapper, M. H. (1996). One-electron oxidation reactions of some purine and pyrimidine bases in aqueous solutions. electrochemical and pulse radiolysis studies. J. Phys. Chem. 100, 14751-14761. doi: 10.1021/jp960590g

Hofsass, R., Sinn, S., Biedermann, F., and Wagenknecht, H. A. (2018). Programmable and sequence-selective supramolecular assembly of two different chromophores along DNA templates. Chem. Eur. J. 24, 16257-16261. doi: 10.1002/chem.201804314

Ishutkina, M. V., Berry, A. R., Hussain, R., Khelevina, O. G., Siligardi, G., and Stulz, E. (2018). Self-assembled porphyrazine nucleosides on DNA templates: highly fluorescent chromophore arrays and sizing forensic tandem repeat sequences. Eur. J. Org. Chem. 2018, 5054-5059. doi: 10.1002/ejoc.201800683

Kallenbach, N. R., Ma, R.-I., and Seeman, N. C. (1983). An immobile nucleic acid junction constructed from oligonucleotides. Nature 305, 829-831. doi: $10.1038 / 305829 \mathrm{a} 0$

Kato, T., Kashida, H., Kishida, H., Yada, H., Okamoto, H., and Asanuma, H. (2013). Development of a robust model system of FRET using base surrogates tethering fluorophores for strict control of their position and orientation within DNA duplex. J. Am. Chem. Soc. 135, 741-750. doi: 10.1021/ja309279w

Kawasumi, K., Mochida, K., Segawa, Y., and Itami, K. (2013). Palladiumcatalyzed direct phenylation of perylene: struvtural and optical properties of 3,4,9-triphenylperylene and 3,4,9,10-tetraphenylperylene. Tetrahedron 69, 4371-4374. doi: 10.1016/j.tet.2013.01.037

Kumar, C. V., and Duff, M. R. Jr. (2009). DNA-based supramolecular artificial light harvesting complexes. J. Am. Chem. Soc. 131, 16024-16026. doi: 10.1021/ja904551n 
Li, S., Langenegger, S. M., and Häner, R. (2013). Control of aggregationinduced emission by DNA hybridization. Chem. Commun. 49, 5835-5837. doi: $10.1039 / \mathrm{c} 3 \mathrm{cc} 42706 \mathrm{~d}$

Lubrich, D., Lin, J., and Yan, J. (2008). A contractile DNA machine. Angew. Chem. Int. Ed. 47, 7026-7028. doi: 10.1002/anie.200800476

Probst, M., Langenegger, S. M., and Häner, R. (2014). A modular LHC built on the DNA three-way junction. Chem. Commun. 50, 159-161. doi: $10.1039 / \mathrm{C} 3 \mathrm{CC} 47490 \mathrm{~A}$

Rossetti, L., Franceschin, M., Schirripa, S., Bianco, A., Ortaggi, G., and Savino, M. (2005). Selective interactions of perylene derivatives having different side chains with inter- and intramolecular G-quadruplex DNA structures. A correlation with telomerase inhibition. Bioorg. Med. Chem. Lett. 2005, 413-420. doi: 10.1016/j.bmcl.2004.10.061

Rothemund, P. W. (2006). Folding DNA to create nanoscale shapes and patterns. Nature 440, 297-302. doi: 10.1038/nature04586

Ruiz-Carretero, A., Janssen, P. G. A., Stevens, A. L., Surin, M., Herz, L. M., and Schenning, A. P. H. J. (2011). Directing energy transfer in discrete one-dimensional oligonucleotide-templated assemblies. Chem. Commun. 47, 884-886. doi: 10.1039/C0CC04128A

Sargsyan, G., Leonard, B. M., Kubelka, J., and Balaz, M. (2014). Supramolecular ssDNA templated porphyrin and metalloporphyrin nanoassemblies with tunable helicity. Chem. Eur. J. 20, 1878-1892. doi: 10.1002/chem.201 304153

Seeman, N. C. (1982). Nucleic acid junctions and lattices. J. Theor. Biol. 99, 237-247. doi: 10.1016/0022-5193(82)90002-9

Stevens, A. L., Janssen, P. G. A., Ruiz-Carretero, A., Surin, M., Schenning, A. P. H. J., and Herz, L. M. (2011). Energy transfer in single-stranded DNA-templated stacks of naphthalene chromophores. J. Phys. Chem. C 115, 10550-10560. doi: $10.1021 /$ jp2036444
Stockert, A. L., Shinde, S. S., Anderson, R. F., and Hille, R. (2002). The reaction mechanism of xanthine oxidase: evidence for two-electron chemistry rather than sequential one-electron steps. J. Am. Chem. Soc. 124, 14554-14555. doi: $10.1021 /$ ja027388d

Teo, Y. N., Wilson, J. N., and Kool, E. T. (2009). Polyfluorophores on a DNA backbone: a multicolor set of labels excited at one wavelength. J. Am. Chem. Soc. 131, 3923-3933. doi: 10.1021/ja805502k

Trifonov, A., Raytchev, M., Buchvarov, I., Rist, M., Barbaric, J., Wagenknecht, H. A., et al. (2005). Ultrafast energy transfer and structural dynamics in DNA. J. Phys. Chem. B 109, 19490-19495. doi: 10.1021/jp052108c

Varghese, R., and Wagenknecht, H. A. (2009). White-light-emitting DNA (WED). Chem. Eur. J. 15, 9307-9310. doi: 10.1002/chem.200901147

Wang, R., Liu, W., and Seeman, N. C. (2009). Prototyping nanorod control: a DNA double helix sheathed within a DNA six-helix bundle. Chem. Biol. 16, 862-867. doi: 10.1016/j.chembiol.2009.07.008

Yakovchuk, P., Protozanova, E., and Frank-Kamenetskii, M. D. (2006). Basestacking and base-pairing contributions into thermal stability of the DNA double helix. Nucl. Acids Res. 34, 564-574. doi: 10.1093/nar/gkj454

Conflict of Interest: The authors declare that the research was conducted in the absence of any commercial or financial relationships that could be construed as a potential conflict of interest.

Copyright $(2019$ Fritz and Wagenknecht. This is an open-access article distributed under the terms of the Creative Commons Attribution License (CC BY). The use, distribution or reproduction in other forums is permitted, provided the original author(s) and the copyright owner(s) are credited and that the original publication in this journal is cited, in accordance with accepted academic practice. No use, distribution or reproduction is permitted which does not comply with these terms. 